\title{
Ensino e aprendizagem impulsionados pelo pensamento computacional
}

\author{
Teaching and learning powered by computational thinking \\ Enseñanza y aprendizage impulsados por el pensamiento computacional
}

\author{
Karine Serpa Franco \\ ORCID: https://orcid.org/0000-0002-3829-5823 \\ Universidade Federal Fluminense, Brasil \\ E-mail: karinesfranco.edu@gmail.com \\ Alessandra Teles Sirvinskas Ferreira \\ ORCID: https://orcid.org/0000-0002-2347-815X \\ Universidade Federal Fluminense, Brasil \\ E-mail: atsf15@gmail.com \\ Lucianne Fragel Madeira \\ ORCID: https://orcid.org/0000-0001-6747-2828 \\ Universidade Federal Fluminense, Brasil \\ E-mail:lfragel@id.uff.br \\ Suzete Araujo Oliveira Gomes \\ ORCID: https://orcid.org/0000-0001-7130-8254 \\ Universidade Federal Fluminense, Brasil \\ E-mail: suzetearaujo@id.uff.br \\ Sergio Crespo Coelho da Silva Pinto \\ ORCID: https://orcid.org/0000-0001-6914-2398 \\ Universidade Federal Fluminense, Brasil \\ E-mail: screspo@id.uff.br
}

\begin{abstract}
Resumo
Pensamento Computacional (PC) é uma estratégia utilizada no processo ensino/aprendizagem, envolvendo resolução de problemas, de forma a desenvolver o pensamento crítico e criativo, de forma autônoma, interdisciplinarmente. O presente artigo apresenta uma análise reflexiva sobre o tema, fundamentando-se em literaturas que abordam o PC e seus desdobramentos. Tem como objetivo discutir os benefícios decorrentes do desenvolvimento do PC na educação básica, bem como descrever brevemente algumas estratégias já em uso para que o PC possa ser ensinado, beneficiando, assim, alunos, professores e a sociedade. Concluímos que ao desenvolver o PC o aluno passa a ser sujeito construtor de conhecimento, de forma crítica, criativa, polivalente, flexível e inovadora, e que isso pode se dar através de estratégias diversas, seja pelo ensino da robótica, programação em blocos, narrativas digitais, games, com atividades sem o uso de tecnologias, ou de forma transversal dentro das disciplinas.
\end{abstract}

Palavras-chave: Aprendizagem; Ensino; Pensamento computacional.

\begin{abstract}
Computational Thinking (CT) is a strategy used in the teaching/learning process, involving problem solving, in order to develop critical and creative thinking, autonomously, interdisciplinaryly. This article presents a reflexive analysis on the theme, based on literatures that address CT and its consequences. It aims to discuss the benefits of the development of CT in basic education, as well as briefly describe some strategies already in use so that the CT can be taught, thus benefiting students, teachers and society. We conclude that when developing the CT the student becomes a subject constructor of knowledge, in a critical, creative, versatile, flexible and innovative way, and that this can happen through various strategies, whether through the teaching of robotics, block programming, digital narratives, games, with activities without the use of technologies, or in a transversal way within the disciplines.
\end{abstract}

Keywords: Learning; Teaching; Computational thinking.

\section{Resumen}

El Pensamiento Computacional (PC) es una estrategia utilizada en el proceso de enseñanza/aprendizaje, que implica la resolución de problemas, con el fin de desarrollar el pensamiento crítico y creativo, de forma autónoma, interdisciplinaria. Este artículo presenta un análisis reflexivo sobre el tema, basado en literaturas que abordan el PC y sus consecuencias. Su objetivo es discutir los beneficios del desarrollo del PC en la educación básica, así como describir brevemente algunas estrategias ya en uso para que el PC pueda ser enseñado, beneficiando así a los estudiantes, los profesores y la sociedad. Concluimos que al desarrollar el PC el estudiante se convierte en una asignatura constructora de conocimiento, de una manera crítica, creativa, versátil, flexible e innovadora, y que esto puede suceder a través de diversas estrategias, ya sea a través de la enseñanza de la robótica, la programación de 
bloques, narrativas digitales, juegos, con actividades sin el uso de tecnologías, o de manera transversal dentro de las disciplinas.

Palabras clave: Aprendizaje; Enseñanza; Pensamiento computacional.

\section{Introdução}

Na sociedade contemporânea em que vivemos, as tecnologias estão presentes em nossas vidas cotidianamente. Em se tratando do contexto educacional, nas últimas décadas houve uma ascensão das inovações em tecnologia com o objetivo de corroborar com o processo ensino/aprendizagem. Muitos aplicativos para dispositivos móveis, softwares, programas e plataformas foram criadas para estimular e desenvolver habilidades cognitivas nos educandos. A Indústria $4.0^{1}$ com seus sistemas ciber-físicos conectados, imprimem um 1 novo ritmo a formação do profissional. Este novo perfil vem adornado de requisitos nem sempre explorados nos bancos escolares. Pertencer a este novo mundo tecnológico exige um aluno mais reflexivo, mais criativo, mais sistêmico na busca de algumas soluções para seus problemas. Podemos perceber que os novos estudantes possuem uma facilidade maior com os artefatos tecnológicos, porém esta fluência tecnológica de "ser usuário" não satisfaz a necessidade de "ser criativo". Sendo assim, é interessante que novas estratégias de aprendizado ativo possam ser pensadas e refletidas no sentido de como potencializar o ser criativo, inventivo e reflexivo no corpo discente.

Estudos no campo da Informática da Educação reforçam que não basta apenas aprender a utilizar tais ferramentas tecnológicas, de forma aleatória. Mas sim, como essas ferramentas contribuirão para que os alunos vivam em sociedade, se apropriando das tecnologias existentes e tendo uma visão crítica sobre o mundo que os cercam.

Para tanto, torna-se imprescindível entender como aprendendo essas habilidades, os alunos se constituirão como sujeitos ativos na construção do conhecimento, entendo o contexto tecnológico que os cercam. E o Pensamento Computacional traz justamente a ideia de compreender as situações propostas e criar soluções, de forma criativa e autônoma.

No texto da Base Nacional Comum Curricular (BNCC) há muitas menções do termo PC. O conceito é atrelado ao ensino da matemática, principalmente, e também nas competências gerais propostas:

Compreender, utilizar e criar tecnologias digitais de informação e comunicação de forma crítica, significativa, reflexiva e ética nas diversas práticas sociais (incluindo as escolares) para se comunicar, acessar e disseminar informações, produzir conhecimentos, resolver problemas e exercer protagonismo e autoria na vida pessoal e coletiva (Brasil, 2017, p. 9).

O presente ensaio tem como objetivo discutir os benefícios decorrentes do desenvolvimento do Pensamento Computacional na educação básica, bem como descrever brevemente algumas estratégias já em uso para que o PC possa ser ensinado, beneficiando, assim, alunos, professores e a sociedade.

\section{Referencial: O Pensamento Computacional}

Pensamento Computacional (PC) é um termo cunhado por Wing (2006), em que descreve como sendo a habilidade de pensar em vários níveis de abstração, de forma sistemática, enxergando a inteireza, a complexidade do todo daquilo que se precisa resolver. Porém, tendo a capacidade de fragmentá-lo em partes tais que permitam sua resolução, fazendo uso de forma eficiente dos recursos tecnológicos para alcançar a resolução do problema apresentado. Todavia, Valente (2016) relata que ainda há muita discussão quanto a essa definição e, mesmo após diversos debates os especialistas na área da computação não conseguiram chegar a um acordo.

\footnotetext{
${ }^{1}$ A Indústria 4.0 é um fenômeno global, iniciado na Alemanha, revolucionando o conceito e métodos de trabalho através da criação de fábricas inteligentes que integram sistemas de automação, sistemas ciber-físicos e internet. Possui como agentes facilitadores para o fenômeno da globalização a Tecnologia da Informação, a existência de uma fonte unificada de dados consistentes, a autonomia e a cooperação (Lima \& Pinto, 2019).
} 
Vale destacar um outro conceito de PC, dado pela Royal Society (2012 apud Nascimento et al., 2018), "o Pensamento Computacional é o processo de reconhecer os aspectos da computação no mundo que nos rodeia e aplicar as ferramentas e técnicas da Ciência da Computação para entender e raciocinar sobre os sistemas e processos naturais e artificiais" (p.29), baseado na premissa que, fora dos muros dos laboratórios, o mundo que nos cerca está impregnado de conceitos relacionados à Ciência da Computação e que necessitamos reconhecer a aplicar seus aspectos e funcionalidades.

Embora haja toda essa divergência, um aspecto que todos concordam é de que o PC tem muito a contribuir para o desenvolvimento do processo de raciocínio dos alunos e que deve estar presente nas escolas. Tal implementação já pode ser observada em alguns países como EUA, Nova Alemanha, Argentina, Canadá (Werlich et al., 2018), Nova Zelândia, Inglaterra, Lituânia, Holanda, Suécia, Portugal, Grécia, Irlanda, Dinamarca, Finlândia, Itália e no Brasil (Valente, 2016). Alguns trabalham de forma transversal, dentro de outras disciplinas nas escolas, outros desenvolveram disciplinas específicas de robótica e/ou de programação. Valente (2016) considera, após suas pesquisas, que o trabalho transversal é mais adequado, envolvendo o PC em todas as disciplinas.

Mitchel Resnick (2014), um dos criadores do programa Scratch, apresenta sua tese de que a educação está em um processo de evolução e que a habilidade de programar é útil para o aprendizado, compara essa à habilidade de ler e escrever. Justifica essa comparação ao afirmar que no processo de aprender a programar desenvolve-se diversas capacidades/habilidades e se aprende através da programação. Dentre estas capacidades/habilidades ele cita: a capacidade de organizar ideias, o raciocínio sistemático, a criatividade, a capacidade de projetar, de planejar, a capacidade de criar soluções para problemas apresentados, a habilidade de fragmentar um problema para melhor solucioná-lo, desenvolve o trabalho colaborativo através do compartilhamento de ideias e projetos, a habilidade de construir e compreender conceitos, a capacidade de testar e depurar, de reutilizar e remixar, de perseverar, a participação ativa e consciente.

Para corroborar essa afirmativa ele apresenta diversos projetos desenvolvidos por crianças e adultos ao utilizarem o Scratch, um software gratuito de programação desenvolvido por ele junto com sua equipe de pesquisadores. De forma leve e bem exemplificada ele apresenta temas diversos que foram desenvolvidos no processo de criação desses projetos e, como o interesse dos envolvidos podia conduzi-los a uma busca mais aprofundada por novos conhecimentos com o intuito de aprimorarem seus projetos ou de conseguirem concretizar aquilo que idealizaram em suas mentes (Pereira \& Sampaio, 2008; Sápiras et al., 2015; Conceição \& Vasconcelos, 2018).

Resnick (2014) afirma que desenvolve-se um aprendizado marcante pois é significativo e construído na prática. Destaca a diferença entre ser um uma pessoa que interage com a tecnologia e uma pessoa cria, comparando as pessoas que apenas interagem com as pessoas que conseguem ler mas não sabem escrever, elas consomem mas não produzem, não há fluência. A fluência tecnológica traz benefícios, os quais podem ser observados nos países que já estão inserindo esse conteúdo em seus currículos, seja como disciplina específica quanto como conteúdo transversal.

\section{Metodologia}

$\mathrm{O}$ artigo apresenta uma análise reflexiva sobre o tema, fundamentando-se em literaturas que abordam o Pensamento Computacional (PC) e seus desdobramentos. Os artigos tratam das estratégias de utilização do PC no Ensino Fundamental, no Ensino Médio, no ensino da matemática, por meio dos Objetos de Aprendizagem (OAs), de forma Interdisciplinar e no Currículo. E a partir das discussões, tratam a importância da formação dos professores para desenvolver atividades voltadas para a apreensão das habilidades e competências do PC, bem como a forma de avaliação do desenvolvimento do PC no aluno.

$\mathrm{O}$ estudo apresenta caráter qualitativo. A pesquisa qualitativa ressalta a importância da forma como o pesquisador interpreta e cria hipóteses a respeito do fenômeno em questão (Koche, 2011). Em geral, ocorre em ambiente natural, o pesquisador é o condutor do estudo, os dados coletados são descritivos, utiliza o método indutivo, o foco do processo é o 
fenômeno em si e a visão e vivência dos sujeitos envolvidos (Ludke \& André, 2013; Pereira et al., 2018).

\section{Resultados e Discussão}

\subsection{Pensamento Computacional: benefícios}

Entende-se que algumas bases são necessárias para um bom desenvolvimento no processo de aprendizagem. Uma boa alfabetização, leitura e escrita, por exemplo, permitem uma fácil compreensão de textos e, portanto, maior facilidade em buscar informações e compreendê-las. Assim como uma boa base dos cálculos matemáticos conduzem a maior facilidade em compreender a aplicação, uso e resolução de fórmulas matemáticas, medidas e cálculos mais complexos. Para Wing (2006), o PC deve ser visto como base essencial para o desenvolvimento do raciocínio lógico e sistematizado no processo de resolução de problemas, levando em consideração a compreensão do comportamento humano. Para a autora este aluno deve ser estimulado desde cedo, assim como a escrita, a leitura e a aritmética. Valente (2016) aponta Papert (1980) como o precursor no esclarecimento quanto ao fato da computação contribuir no processo de pensar, o qual afirmava que "os computadores deveriam ser utilizados para que as pessoas pudessem 'pensar com' as máquinas e 'pensar sobre' o próprio pensar." (Valente, 2016, p. 869).

Valente (2016, p. 871) explica que há um "ciclo de ações descrição-execução-reflexão-depuração" durante qualquer processo de criação de um programa. Primeiro o aluno cria uma ideia de resolução do problema; em seguida a descreve no programa do computador; logo após a máquina (computador) executa os comandos; o aluno então reflete sobre os dados observados e, diante do alcance ou não do objetivo, o aluno decide se será necessária uma nova estratégia ou ação: isso é a depuração. Esse ciclo, Ciclo de Avaliação Construcionista, funciona como uma espiral de aprendizagem, em que o aluno repete a ação mas com o pensamento, o raciocínio modificado diante dos resultados obtidos no ciclo anterior. Sempre há um aprendizado e uma construção de pensamento.

Para Jean Piaget (Pinto \& Nascimento, 2018) o aprender fazendo é o caminho para a construção do conhecimento e é exatamente essa a proposta do PC: o aluno deve elaborar hipóteses e teorias, tentar aplicá-las, analisar os resultados obtidos, modificar o que considerar necessário para atingir seu objetivo final, criar, desenvolver e aplicar de novo. Isso tudo fazendo uso de equipamentos tecnológicos. O aluno é estimulado a ser criativo na busca de soluções de problemas e/ou de elaboração/construção de jogos, aplicativos, games. Portanto, o PC contribui não só para criatividade, mas para a elaboração do pensamento, de forma sistemática, moderna e fazendo uso dos equipamentos tecnológicos de maneira eficaz por parte do aluno. De acordo com Pinto e Nascimento (2018), isso já foi verificado por pesquisadores como Cuny, Snyder e Wing (2010), Barr e Stephenson (2011), Brennan e Resnick (2012).

Ao utilizar o PC em sua prática de ensino, o professor deve elaborar questões que conduzam o aluno a busca por uma solução. Isso significa que há diversas possibilidades a serem exploradas e descobertas pelo aluno, sem que a ele seja imposto um único meio de resolver as questões. Significa também que, não é necessariamente obrigatório ter o nome dos conceitos decorados, há meios de conseguir que o indivíduo se aproprie da base de um conceito pela experimentação, para só depois ser dado a ele o nome daquilo que foi desenvolvido por ele (Pinto \& Nascimento, 2018).

Dentro do ensino de programação é importante que os professores elaborem enunciados contextualizados, dentro de uma realidade prática, e que possam ser corretamente compreendidos pelo aluno. Nesse sentido, ferramentas e métodos que utilizam conceitos de programação, associadas à práticas pedagógicas que estimulem o PC, acabam por desenvolver habilidades para auxiliar os alunos na resolução de problemas, conceber sistemas e compreender o comportamento humano. De acordo com Blikstein (2008), o ensino da programação aumenta a produtividade, inventividade e criatividade. Inspirado em conceitos da Ciência da Computação, traz como benefício a compreensão das situações propostas e a construção de soluções através de modelos matemáticos, científicos ou sociais (Mestre et al., 2015). 
Pinto e Nascimento (2018) afirmam que essa forma de pensar sistematizada, buscando a solução de um problema retirado do contexto do indivíduo, um problema que atende uma demanda local, real e pessoal, o fornecimento de ferramentas para a solução e a aplicação real desta solução, contribui para o aprendizado de abstrações, da criatividade e do raciocínio lógico, do encadeamento de ideias. E que esse tipo de ensino/aprendizagem é motivante, estimulante e atende às demandas do mercado tecnológico atual.

São elencadas, por Pinto e Nascimento (2018) e por Valente (2016), cerca de nove habilidades que podem ser desenvolvidas ao utilizar o PC dentro das práticas pedagógicas, são elas: coleção de dados de forma adequada; análise de dados com sentido, padronização permitindo chegar a conclusões; representação de dados de forma adequada e variada; decomposição do problema tornando-o mais fácil de gerenciar; abstração; algoritmos e procedimentos em que se consegue organizar uma sequência de ações que permitam atingir o objetivo de forma mais eficiente; automação ao saber utilizar os computadores e/ou máquinas para realizarem as tarefas repetitivas e tediosas; simulação ao desenvolver modelos ou representações para averiguar suas hipóteses; paralelização, em que se consegue simultaneamente realizar múltiplas tarefas de forma organizada com o intuito de atingir a meta pretendida.

Ao utilizar o PC, o aluno aprende essas habilidades supracitadas e, paralelamente, desenvolve sua autoconfiança, autonomia, persistência, tolerância, comunicação e capacidade de trabalhar em equipe (Seehorn et al. 2011 apud Pinto \& Nascimento, 2018).

Vale ressaltar como benefício, que o PC não só introduz conceitos e habilidades da Ciência da Computação aos alunos, mas contribui para que obtenham um melhor desempenho em outras áreas, em outras disciplinas (Nascimento et al. 2018).

Nesse sentido, pensando numa visão transformadora da educação, na busca de uma formação voltada ao desenvolvimento humano, o PC estimula muitas habilidades nos alunos, como a proatividade, o autoconhecimento, a autonomia, a criticidade, a resolução de problemas enfrentados no seu cotidiano, bem como envolve aspectos técnicos e cognitivos.

Para toda essa construção do conhecimento, faz-se necessário a utilização de metodologias inovadoras de ensino, que atendam tanto às expectativas do desenvolvimento pessoal dos alunos, quanto às demandas do mercado por profissionais mais qualificados.

A educação deve se preparar para uma reconfiguração de tempo e espaço de aprendizagem, de forma coletiva e colaborativa que estimulem as produções mútuas e a troca recíproca de informações. Para tanto, requer metodologias ativas, ferramentas digitais e/ou analógicas de sala de aula e instituições de ensino que repensem seus processos de ensino e aprendizagem. Fazem parte dessa reconfiguração a interdisciplinaridade, a transdisciplinaridade, a Inteligência Artificial, um currículo contextualizado e flexível.

Assim como o PC, as metodologias ativas através do ensino híbrido, cursos invertidos, cultura do fazer (maker), aprendizagem baseadas em projetos, em redes sociais e aprender ensinando, corroboram com o desenvolvimento da autonomia, criatividade e resiliência (Berbel, 1998).

O cenário atual da Indústria 4.0 ou Quarta Revolução Industrial, caracterizada pelo uso de tecnologias para automação e troca de dados, otimizando a fabricação de produtos e, personalizando os produtos de acordo com a demanda, reforça que o espaço educacional necessita de novas estratégias e metodologias de aprendizagem para a preparação de seus alunos para o mercado de trabalho, com um novo papel para o professor e uma ressignificação do processo de ensino e aprendizagem. As práticas pedagógicas inovadoras, incluindo as metodologias ativas e o PC, tem como uma de suas diretrizes, desenvolver competências e habilidades que vão além do simples domínio técnico, para futuros profissionais que atuarão na Indústria 4.0 (Lima \& Pinto, 2019). 


\subsection{Ferramentas e Estratégias}

Para cada etapa da Educação Básica, de forma gradual, o PC desenvolve habilidades de compreender, definir, modelar, comparar, solucionar, automatizar e analisar problemas e suas soluções. Ensinar processos de informação, para analisar dados e chegar à automação de soluções no cotidiano, prevê ferramentas de transformação do mundo.

De acordo com a Sociedade Brasileira de Computação (SBC, 2019), nos anos iniciais do Ensino Fundamental (EF), o PC desenvolve habilidades e conhecimentos acerca da análise crítica e da argumentação, baseando-se na abstração da informação, dos processos e da resolução de problemas. Nos anos finais (EF), trabalha a automação de dados e programas para alcançar a solução do problema. No Ensino Médio, o foco do PC é na aquisição de técnicas de transformação do problema e na programação (meta programação), através principalmente da Inteligência Artificial (IA) e da robótica, bem como na compreensão e distinção da relação homem-máquina.

Nesse sentido, a partir da compreensão plena do mundo, o aluno aumenta sua capacidade de aprendizagem e de resolução de problemas, de forma interdisciplinar. E será através de experiências concretas que conseguirá construir modelos mentais para a abstração computacional e a aquisição das linguagens de programação.

Para que o PC esteja presente no processo ensino-aprendizagem, faz-se necessária uma intencionalidade, um planejamento das ações, ferramentas e dos recursos para guiar a prática pedagógica e contribuir na formação de alunos mais autônomos, criativos, resilientes e críticos. Embora as vantagens sejam evidentes, cabe questionar: de que forma aplicar?

Com o intuito de estimular a aprendizagem dos alunos, uma das práticas pedagógicas embasadas pelo PC, como ferramentas educativas são conhecidas como Objetos de Aprendizagens (OAs).

Segundo Braga e Menezes (2014), os OAs consistem na reusabilidade em diferentes contextos e funcionam como mediadores do conhecimento. As atividades dos OAs devem ser usadas com a finalidade de desenvolver a cognição, e, neste sentido, precisam ser cuidadosamente planejadas para que a partir delas seja possível construir conhecimentos, desenvolver capacidades, habilidades e competências. Ao utilizar os OAs o aluno se torna o autor e coautor da construção de seu conhecimento.

A aplicação do PC com a utilização de OAs no cenário educacional brasileiro, ocorre em diversas situações e áreas do conhecimento. Os OAs utilizados, de acordo com Nascimento et al. (2018), podem ser concretos (atividades lúdicas para estimular o raciocínio lógico) e conceituais (através da programação). Como exemplos de OAs associadas ao PC, podemos citar: jogos manuais, jogos digitais, Plataforma Code, Scratch, Lego Mindforms, Arduino, Inventor for Androids, dentre outros. Valente (2016) apresenta algumas estratégias que já vêm sendo aplicadas:

Atividades que não usam das tecnologias (Grover \& Pea, 2013 apud Valente, 2016). Neste caso não se usam equipamentos eletrônicos, mas buscam ensinar como um cientista da computação pensa e desenvolve seu raciocínio. Para isso, se utiliza de jogos e competições. Embora seja uma estratégia mais fácil de ser aplicada por não demandar recursos tecnológicos, nem sempre disponíveis nas escolas, há críticas a esta estratégia por não proporcionar ao educando uma aproximação das vivências tecnológicas digitais;

Programação em blocos. Os comandos não precisam ser digitados em formato de códigos. Eles estão disponíveis em blocos que o aluno apenas precisa escolher e ordenar de acordo com o objetivo por ele almejado, isso pode ser feito através de programas como o Scratch (Resnick, 2014), o Snap! e o Alice (Maninila et al., 2014 apud Valente, 2016) que permitem criar "histórias animadas, jogos e apresentações interativas";

Robótica pedagógica. O aluno aprende a programar o computador para obedecer a determinados comandos, ou série de comandos estando plugado ao computador ou desplugado (Valente, 2016).

E segundo Rosa e Coelho Neto (2020): 
Para o desenvolvimento do pensamento computacional por meio do ensino de robótica educacional, constata-se que, cada vez mais um número maior de pesquisadores adotam alguns dos subsídios próprios do design thinking para desenvolver diferentes habilidades durante a realização de suas atividades pedagógicas de robótica educacional. Nesse sentido, acabam por obterem resultados cada vez mais significativos da aprendizagem e, ainda, propiciam a motivação e o interesse de ambos os sexos para a busca de uma formação em áreas científicas (p.19).

Design Thinking. É uma processo de solução de problemas que parte de habilidades mentais complexas desenvolvidas, auxiliando os sujeitos a alcançar soluções inovadoras, empreendedoras para seus problemas e suas demandas (Goldman \& Kabayadondo, 2017);

Narrativas digitais. Permitem que o aluno crie ou reconte uma história através de "imagens, animação, vídeos e sons" (Valente, 2016, p. 876);

Criação de games (Salen \& Zimmerman, 2003 apud Valente, 2016). O aluno precisa desenvolver os desenhos dos personagens; realizar a escolha de músicas e sons; estabelecer as cores; criar uma narrativa e estabelecer como serão as regras e objetivos do jogo, bem como selecionar quais softwares e dispositivos serão necessários para acessar e jogar.

A gamificação e o PC, para Gomes et al. (2020):

A utilização de um jogo virtual apropriado para facilitar o ensino pode fornecer ao aluno um ambiente lúdico que utiliza tecnologias de modo estimulante, promovendo a familiaridade e tirando o foco do quadro e do professor, criando condições favoráveis para que o aluno procure ser protagonista no desenvolvimento de sua base de conhecimentos e aprenda a relacionar e aplicar os conceitos da área de computação (p.4).

Uso de simulações. O aluno precisa escolher um fenômeno a ser observado, inserir as variáveis e analisar os resultados observados, "com isto, ele pode construir a representação matemática do fenômeno" (Valente, 2016, p. 878).

Os OAs são de grande relevância para o desenvolvimento do PC, à medida que estimulam a aprendizagem, a construção do conhecimento, a um interesse maior pelo conteúdo proposto pelo professor, pelo fato de ser lúdico e significativo para os alunos.

\section{Considerações Finais}

Entendemos, portanto, que o Pensamento Computacional consiste em associar o pensamento crítico aos fundamentos da Ciência da Computação, por meio de abstração e decomposição de idéias e atitudes, de forma sistemática, sabendo fazer uso adequado dos recursos tecnológicos, na resolução de problemas,

A International Labour Organization (2019) aponta o surgimento de algumas profissões no mercado de trabalho atual, tais como especialista em cloud computer, técnicos digitais, cientista de dados, especialista em inteligência artificial, programadores; entendendo que as atividades de baixa complexidade passarão, cada vez mais, a serem executadas pelas máquinas e as mais complexas são as que o ser humano deve assumir de forma polivalente, flexível e inovadora.

Diante desse cenário, concluímos que torna-se imprescindível desenvolver o PC no aluno, passa este a ser sujeito construtor de conhecimento, de forma crítica, criativa, polivalente, flexível e inovadora, e que isso pode se dar através de estratégias diversas, seja pelo ensino da robótica, programação em blocos, narrativas digitais, games, com atividades sem o uso de tecnologias, e pela utilização de tais ferramentas nas mais diversas áreas do conhecimento, de forma interdisciplinar.

De acordo com os artigos analisados, no Brasil a discussão sobre o PC como ferramenta de aprendizagem significativa, pertencente ao currículo e às práticas pedagógicas, ainda é muito recente. Ainda há muito o que se fazer no contexto da educação brasileira para garantir a efetivação de práticas e metodologias embasadas no PC. 
Nesse sentido, pensar em estratégias de aprendizagem voltadas para um pensamento criativo, embebido de significados, de forma colaborativa, inter e transdisciplinar, tanto na escola, como fora dela, em diferentes contextos, físico e/ou virtual, sem dúvida seria um grande passo a ser dado na qualidade da educação brasileira.

Como possibilidades para novas pesquisas na relação do PC com o processo ensino e aprendizagem, sugerimos estudos de casos comparativos em diferentes regiões brasileiras, levando em consideração sua cultura, seus valores e de que forma os alunos utilizaram-se dessas ferramentas pedagógicas para solucionar problemas inerentes ao ambiente em que vivem.

\section{Referências}

Barcelos, T., Muñoz, R., Villaroel, R. \& Sibeira, I. (2015). Relações entre o Pensamento Computacional e a matemática: uma revisão sistemática da literatura. Anais dos Workshops do IV Congresso Brasileiro de Informática na Eduacação (WCBIE).

Berbel, N. A. N. (1998). A problematização e a aprendizagem baseada em problemas:diferentes termos ou diferentes caminhos? Interface - Comunicação, Saúde, Educação. 2(2), 139-54. http://www.scielo.br/pdf/icse/v2n2/08.

Blikstein, P.(2008). O Pensamento Computacional e a Reinvenção do Computador na Educação. http://bit.ly/11XlbNn>.

Bordini, A., Avila, C., Cunha, M., Cavalheiros, S. \& Foss, L. (2017). Pensamento Computacional no Ensino Fundamental e Médio: uma revisão sistemática. Anais do XXVIII Simpósio do Congresso Brasileiro de Informática na Educação (SBIE).

Braga, J. \& Menezes, L. (2014). Introdução aos Objetos de Aprendizagem. In: Braga, J. C. (Org). Objetos de Aprendizagem - Volume 1: Introdução e Fundamentos. Editora da UFABC.

Brasil. (2017) Base Nacional Comum Curricular - BNCC. MEC.

Carvalho, J. M. de, Netto, J. F. \& Almeida, T. O. (2017). Revisão sistemática de literatura sobre o Pensamento Computacional por Meio de Objetos de Aprendizagem. Anais do XXVIII Simpósio de Informática na Educação - Simpósio Brasileiro de Informática na Educação (SBIE).

Conceição, J. H. C. \& Vasconcelos S. M. (2018). Jogos digitais no ensino de ciências: contribuição da ferramenta de programação scratch. Areté Manaus, 11 (24), 170-185.

Goldman, S. \& Kabayadondo, Z. (2017). Talking design thinking to school: How the Technology of Design can transform teachers, learners, and classrooms. Routledge: New York.

Gomes, O. de S. M., Rodrigues, W. de A. \& Franco, R. A. S. R. (2020). Gamificação e pensamento computacional: análise de uma experiência no ensino médio / técnico-educacional. Pesquisa, Sociedade e Desenvolvimento, 9, e543910805. 10.33448/rsd-v9i10.8053. https://rsdjournal.org/index.php/rsd/ar ticle/view/8053.

International Labour Organization (2019). Work for a brighter future. Global Commission on the Future of Work. International Labour Office. https://drive.google.com/file/d/1SKkO_KeA40fX29ohyMHDs2EeWdH_B_TA/view.

Koche, J. C. (2011). Fundamentos de metodologia científica. Vozes.

Ludke, M. \& Andre, M. E. D. A. (2013). Pesquisa em educação: uma abordagem qualitativa. (2a ed.), EPU.

Mestre, P., Andrade, W., Guerrero, D., Sampaio, L., Rodrigues, R. da S. \& Costa, E. (2015). Pensamento Computacional: Um estudo empírico sobre as questões de matemática do PISA. In: Anais dos Workshops do Congresso Brasileiro de Informática na Educação.

Nascimento, C. A., Santos, D. A. \& Tanzi, A. (2018). Pensamento Computacional e Interdisciplinaridade na Educação Básica: um mapeamento sistemático. In: Anais dos Workshops do VII Congresso Brasileiro de Informática na Educação.

Pereira, A. S. T. \& Sampaio, F. F. (2008). AVITAE: development of a computational modeling system to biology education. Ciências \& Cognição 13 (2): $51-$ 70 .

Pereira A. S., Shitsuka, D. M., Parreira, F. J. \& Shitsuka, R. (2018). Metodologia da pesquisa científica. UFSM. https://repositorio.ufsm.br/bitstream/handle/ 1/15824/Lic_Computacao_Metodologia-Pesquisa-Cientifica.pdf?sequence=1

Pinto, A. G. \& Lima, G. S. (2019). Indústria 4.0: um novo paradigma para a indústria. Interface Tecnológica, 16. 10.31510/infa.v16i2.642.

Pinto, S. C. C. S. \& Nascimento, G. S. R. (2018). O pensamento computacional e a a nova sociedade. In: Valente, J. A., Freire, F. M. P. \& Arantes, F. L. (org.). Tecnologia e educação: passado, presente e o que está por vir. 301-322. https://drive.google.com/file/d/1 wpNjTIZAcsbIQCX-BdMaqLmWKPdsa2U/view.

Resnick, M. (2014). Aprender a programar, programar para aprender. Palestra no evento Transformar 2014. https://www.youtube.com/watch?t ime_continue $=2 \& \mathrm{v}=\mathrm{hRGJUc2opQ} 4 \&$ feature=emb_logo.

Rosa, V. \& Coelho Neto, J. (2020). Design Thinking e Computational Thinking e suas articulações para o ensino de Robótica Educacional: uma revisão. Pesquisa, Sociedade e Desenvolvimento, 9, e6659109019, 2020. 10.33448/rsd-v9i10.9019. https://rsdjournal.org/index.php/rsd/article/view/9019.

Sánchez, J. (org.). ( 2018). Nuevas ideas em Informatica Educativa. 14, 375-384. 
Research, Society and Development, v. 10, n. 10, e402101019099, 2021

(CC BY 4.0) | ISSN 2525-3409 | DOI: http://dx.doi.org/10.33448/rsd-v10i10.19099

Sápiras, F. S., Vecchia, R. D., \& Maltempi, M. V. (2015). Utilização do Scratch em sala de aula. Educação Matemática Pesquisa, 17 (5), 973 - 980

Sociedade Brasileira de Computação. (2019). Diretrizes para ensino de Computação na Educação Básica. 25p. https://www.sbc.org.br/documentos-dasbc/send/203-educacao-basica/1220-bncc-em-itinerario-informativo-computacao-2.

Valente, J. A. (2016). Integração do pensamento computacional no currículo da educação básica:diferentes estratégias usadas e questões de formação de professores e avaliação do aluno. Revista e-Curriculum, 14, 864 - 897. Programa de Pós-graduação Educação: Currículo - PUC/SP. http://revistas.pucsp.br/index.php/curriculum.

Valente, J. A. (2018). Inovação nos processos de ensino e de aprendizagem: o papel das tecnologias digitais. p. 17-41. In: Valene, J. A., Freire, F. M. P. \& Arantes, F. L. (Org.). Tecnologia e educação: passado, presente e o que está por vir - Campinas, SP: NIED/UNICAMP, 406 p.

Werlich, C., Kemczinski, A. \& Gasparini, I. (2018). Pensamento Computacional no Ensino Fundamental: um mapeamento sistemático. In: Sánchez, J. Editor. Nuevas Ideas en Informática Educativa, 14, 375 - 384.

Wing, J. M.(2006). Computational Thinking. Communications of the ACM. 49(3). 